\section{Lymphedema and nutrition: A review}

\author{
Attilio Cavezzi, Simone Ugo Urso, \\ Lorenzo Ambrosini, Sonia Croci, Fausto \\ Campana, Giovanni Mosti
}

Association Nutrage, S. Benedetto del Tronto, Italy

\begin{abstract}
Nutrition is considered a basic component in the management of any vascular disease. Lymphedema is characterised by an increase of interstitial fluid due to a lymphatic system morphological and/or functional alteration. Therapeutic management of lymphedema includes a multi-faceted approach based on compression and physiotherapy mainly. Weight control and antiinflammatory and anti-edema diet are two additional necessary components of the holistic therapy in presence of primary or secondary limb lymphedema. This narrative review provides the available information derived from the scientific literature on nutrition in lymphedema, which anyway lacks robust evidence. Additional information and speculations are provided on the role of food, diet, nutraceuticals and fasting on the basic processes at the root of the chronic progressive degeneration of tissue lymph stasis, i.e. weight excess, inflammation, edema, fibroadiposis. More targeted and randomized studies are needed in order to assess and standardise the obvious, so far neglected, role of nutrition in lymphedema patients.
\end{abstract}

\section{Introduction}

Lymphedema (LYM) is a chronic vascular disease which typically involves lower and/or upper limbs, but can affect different body regions as well. Whichever edema depends upon an overload of a normal lymphatic system or upon a dysfunction of the lymphatic system for an anatomical or functional pathology of the lymphatic system. ${ }^{1}$ In fact recent scientific studies ${ }^{2}$ updated the old microvascular homeostasis concepts which were based on the largely prevailing role of the venous network for the reabsorption of the fluids from the interstice. These studies showed that in most body areas, namely in the extremities, the lymphatic system is responsible for over $80-90 \%$ of the interstitial fluids derived from the physiological homeostasis of the microvascular-tissue unit. By definition edema results from an alteration of natural homeostasis with an accumulation of fluid in the interstitial matrix; consequently whichever vascular and non-vascular origin limb-edema is strictly related to lymphatic system dysfunction, hence to a fluid stasis which is linked to a lymphatic anatomic disease (LYM) or lymphatic functional impairment/overwhelming.

When lymphatic insufficiency is contemplated, it is subdivided into three categories from a pathophysiological point of view: i) dynamic insufficiency, involving high lymph flow, overwhelming the maximum load-output capacity of a physiologically normal lymphatic system; ii) mechanical insufficiency (properly known as LYM), involving low lymph flow due to anatomical alterations in the network of vessels and lymph nodes; iii) safety valve insufficiency, the combined effect of increased lymph flow and the reduced drainage capacity in the diseased lymphatic system. Any microvascular/tissue imbalance invariably results in an altogether of progressive different pathologic phenomena, such as: edema, hypoxia, oxidative stress, inflammation, fibrosis.

LYM is typically characterised by a chronic inflammation of the involved cellular matrix, with a progressive tendency towards a fibrotic transformation of the lymphedematous tissues. ${ }^{1,3}$ More generally the systemic low-grade chronic cellular inflammation (LGCCI) is definitely linked to aging and to multiple chronic degenerative diseases, such as diabetes, metabolic syndrome, atherosclerosis, neurodegeneration, cancer, autoimmunitary diseases. ${ }^{4}$

Among the many factors that trigger edema of the lower and upper limbs (e.g. veno-lymphatic diseases protein dyscrasias, cardio-nefro-hepato-enteropathy, drugs, etc.), wrong eating habits may play a significant role through different pathophysiology mechanisms.

In particular the classic western nutrition, which is primarily based on carbohydrates and refined/processed foods, favours the systemic $\mathrm{LGCCI}^{5}$ and this pathologic process invariably generates and perpetuates any condition of edema in the human body.

Therapeutic management of limb LYM is notoriously of holistic nature, needing a multi-faceted approach ${ }^{1,3}$ based on the possible combination of the following treatments: i) manual lymphatic drainage; ii) compression (bandages, velcro adjustable wraps, elastic garments); iii) electro-medical devices such as intermittent pneumatic compression or bioresonance-microcurrent devices; iv) specific exercising; v) skin care and hygiene rules; vi) drugs/nutraceuticals
Correspondence: Attilio Cavezzi, Association Nutrage, S. Benedetto del Tronto, Italy.

E-mail: info@cavezzi.it

Key words: Nutrition; lymphedema; nutraceuticals; diet; inflammation; overweight; obesity; fasting.

Received for publication: 11 April 2019.

Accepted for publication: 11 April 2019.

This work is licensed under a Creative Commons Attribution 4.0 License (by-nc 4.0).

(C) Copyright A. Cavezzi et al., 2019

Licensee PAGEPress, Italy

Veins and Lymphatics 2019; 8:8220

doi:10.4081/vl.2019.82220

and proper nutrition; vii) surgery in selective cases. Adequate nutrition and proper lifestyle represent two basic beneficial issues for the lymphedematous patients affected by a life-lasting debilitating disease. The influence of nutrition in venous and lymphatic diseases has been highlighted in literature, mainly focusing on leg venous ulcers and varicose veins of the lower limbs. ${ }^{6-8}$ The possible role of nutrition in limb LYM has been limitedly investigated and scarce literature evidence is available on this topic. This narrative review provides an overview on the available literature data regarding nutrition in edema and more specifically in LYM. A literature search was performed through PubMed, Embase, Google Scholar and the Cochrane Library, using the following headings and keywords: chronic edema, lymphedema nutrition and edema, lymphedema treatment, food and edema, diet and edema, oxidative stress and edema, inflammation and lymphedema.

Our aim is to highlight the relationship between nutrition and LYM, providing possible evidence on the beneficial/detrimental effect of proper/inadequate nutrition respectively within LYM management.

\section{Current knowledge on nutrition and lymphedema}

Nutrition studies the correct intake of macronutrients through food. Feeding provides the necessary substances for the physiologic processes of living organisms. More properly, nutrition is the act that is accomplished by taking food on the basis of physical or psychic stimuli. Food consists of three main macronutrients (carbohydrates, lipids and proteins) and micronutrients 
(mineral salts, vitamins). A correct diet is essential for a healthy living and to slow down aging process, within the concept of an adequate metabolism in any pathophysiologic condition, more specifically in the low-grade chronic cellular inflammation which is at the base of any chronic degenerative disease, such as LYM.

LYM is characterised in fact by a progressive increase of inflammation, fat deposition and fibrosis in the edematous tissues. ${ }^{1,3}$ Inflammatory processes in LYM derive from the biochemical nature of the stagnating fluids in the tissues (including the residual, larger macromolecules), which play a major role in the chronic evolution of LYM. ${ }^{9-12}$

The deleterious and increasing cascade of all inflammatory cytokines is recognized as the basic biochemical factor which contributes to LYM worsening and complications. ${ }^{11,12}$ Preliminary studies on the therapeutic possibilities of anti-inflammatory molecules in LYM have been recently reported as well. ${ }^{13}$

Due to the relevant pathogenicity of chronic inflammation in LYM course, the possible positive or negative role of a few pro-inflammatory/anti-inflammatory foods in this field is object of current research. Of interest, systemic lymphatic system is conversely involved in the multi-level regulation of the pathophysiological mechanisms of metabolic syndrome and inflammatory states, ${ }^{14}$ thus confirming the strict interrelationship among inflammation, nutrition, metabolic pathways and the lymphatic system of human body.

Another biochemical relevant issue in LYM patients is their higher levels of oxidative stress in the affected areas. ${ }^{15}$ The imbalance between free radical production and antioxidant systems activity has been proven in lymphatic and venous diseases as well, namely in leg venous ulcers and varicose veins. ${ }^{16-19}$

The role of reactive oxygen species has been elucidated in several chronic diseases, with clear repercussion on the LGCCI. Increased oxidative stress results in a growing inflammatory process, together with the well-recognised accumulation of lipoperoxidation, nucleic acid damages and protein degeneration in limb tissues cells. Several antioxidant natural substances have been tested through nutrition and supplements in vascular diseases, ${ }^{20}$ but no specific beneficial effect has been clearly shown in the vast majority of the trials as to cardiovascular diseases when referring to Vitamin E, C and other similar classes of antioxidants. Conversely polyphenols exhibited a few interesting effects in vascular diseases; they represent a specific class of natural antioxi- dant principles, which showed multiple beneficial actions on the general free radical production, as well as polyphenols elicit the $\mathrm{NrF} 2$ and ARE system pathway acting as xenobiotics in a hormetic fashion. ${ }^{21,22}$

The intake of polyphenols seems to provide a beneficial effect in venous ${ }^{23-25}$ and lymphatic diseases. . $^{1,26-29}$ Namely coumarin/curcumin-rich foods or, better, nutraceuticals seem to target a few of the basic pathologic processes at the root of LYM. ${ }^{26,27,29-31}$ In fact they showed a lymphangion/macrophage-targeted action, as well as a general anti-inflammatory and anti-edema action. Most polyphenols similarly represent a prebiotic food, which regulates the gut microbiota, one of the most innovative target in presence of a chronic degenerative disease. Lastly polyphenols are referred as to epigenetic switches, which are able to activate and deactivate genes with specific beneficial effects on human metabolism and aging.

The major issue, which is related to nutrition in LYM patients, is the overweight/obesity role in the onset and worsening of lymph stasis. Multiple reasons objectively link the presence of overweight or obesity to edema of upper and especially lower extremities.

Overweight or obese patients may have one or more of the following pathophysiologic alterations which strictly relate to limb edema: i) impaired diaphragm function and increased intra-abdominal pressure with dysfunction of fluid cardiopetal aspiration/ drainage; ii) reduced/altered ambulation/ mobility with consequential musculo-vascular limb pump dysfunction and phlebolymphatic stasis; iii) concomitant metabolic syndrome with multiple factors inducing edema (cardiac/renal/liver insufficiency etc.); iv) fluid-retentive adipose tissue in excess; v) hypertension and intake of edema-generating drugs (calcium-blockers, alpha-lytics, second-generation $\beta$-blockers etc.); vi) hormonal alterations such as hyperproduction of insulin and cortisol; vii) skin infections/inflammation which compromise/generate fluid retention furthermore.

The increasing incidence of obesity in western countries' population may potentially increase the rate of LYM, which seems to be strictly related to the body-mass index level. ${ }^{32,33}$ Similar findings have been elicited on the negative impact of obesity upon chronic venous diseases, ${ }^{34}$ which may consequently lead to an increase of phlebolymphedema and venolymphatic incidence worldwide.

Many studies have focused on the negative impact of obesity on lymphatic system, both in terms of LYM onset and in terms of LYM course and prognosis. ${ }^{35,36}$

Lymphoscintigraphic studies have proven significant changes in lymphatic vessels/nodes in obese patients, which may explain the great influence of weight control in LYM patients. Overweight and, more relevantly, obesity proved to be a few of the most significant risk factors for the onset of LYM in patients subjected to cancer-related surgery (especially breast cancer). ${ }^{37}$

Contrasting data were reported by a few authors $^{38}$ who highlighted that breast cancer operated women, whose BMI was $\geq 30$ at the time of operation, were approximately 3.6 times more likely to develop lymphedema at 6 months after diagnosis than subjects with a $\mathrm{BMI}<30(\mathrm{P}=0.007)$. Those with a BMI increase, even above 30, during their first 30 months of survivorship were not more likely to develop secondary LYM than those who did not have similar changes in BMI.

Similarly, when performing treatment of patients with primary or secondary limb LYM, the outcomes are strongly influenced by the weight variations along the time and again overweight, and especially obesity, represent important negative prognostic factors..$^{39,40}$

Proper nutrition, together with adequate physical activity, has represented in the history of medicine and biology the two basic pillars to achieve weight reduction. In fact caloric restriction and dietary intervention on macro-micronutrients may properly address the issue of weight loss in patients affected by upper or lower extremity LYM. Weight reduction in overweight/obese patients proved to be of significant help in reducing both limb edema and LYM-related signs and symptoms. More in detail a systematic review and metanalysis ${ }^{41}$ examined the only two available studies on nutrition and dietary interventions for LYM, indicating that positive effects were found as to LYM volume reduction in both trials. Specifically in upper limb LYM one study demonstrated that caloric reduction for weight loss resulted in a $44 \%$ decrease of volumetry. Probably even higher percentages could be expected in lower limb LYM.

In another study the authors assessed any possible difference between patients with breast-cancer related LYM undergoing a weight reduction diet, or a low-fat diet; they found a significant reduction in body weight, body mass index and skinfold thickness in both groups, compared with controls. Both groups decreased the arm volume in a non-statistically significant way, but both dietary groups showed a significant correlation between weight loss and reduction in arm volume $(\mathrm{P}<0.02){ }^{42}$

The possible influence of a diet includ- 
ing middle chain triglycerides added to conventional complex decongestive treatment was investigated in LYM patients; ${ }^{43}$ the volumetry outcomes showed significant differences between the groups ( $\mathrm{P} \leq 0.05)$, with a greater reduction in the group with the additional specific diet. No difference was reported in skin fold measurements or whole-body water content, whereas the feeling of heaviness in the arms was significantly less in the dietary group.

Similarly the limitation of long chain triglycerides in the diet of LYM patients may induce a significant improvement in volumetry and symptoms, as reported in a preliminary study. ${ }^{44}$

Erroneous nutritional habits invariably lead to some degree of veno-lymphatic function derangement. In case of idiopathic edema of the lower limbs since 1993 a few authors demonstrated that the prevalence of this form of edema was $37.9 \%$ in subjects with eating disorders and $7.4 \%$ in subjects with normal nutrition in terms of eating attitude test outcomes $(\mathrm{P}=0.0001)$. $^{45}$

\section{Possibilities and limitations of nutrition in lymphedema}

Generally foods have been recognized and distinguished as causal factors of body/limb edema or, conversely, antiedema positive adjuvant factors in LYM management.

Fruits, vegetables, fermented foods, garlic, extra-virgin olive oil, nuts and fish represent anti-edemigenous foods, which work by reducing oxidative stress and regulating intestinal bacterial microflora. More specifically extra virgin olive oil is associated with a reduction in cardiovascular disease mortality, as it reduces oxidative stress and inflammation, ${ }^{46-48}$ thanks to its phenolic compounds. ${ }^{47,48}$

The fibers found in fruits and vegetables (e.g. inulin) are rapidly fermented by bacteria that normally reside in the small and large bowel, which leads to the formation of short chain fatty acids, such as acetate, proprionate, butyrate, usually metabolised to produce energy and which exhibit an anti-inflammatory activity ${ }^{49}$ of considerable importance in chronic diseases (LYM included). Fermented products such as kefir also produce an increase in butyrate.

An adequate dietary intake of omega-3, from fish and plant foods (chia seeds, macadamia nuts, walnuts, cashews etc.), namely alpha-linolenic acid or ALA, and eicosapentaenoic and docosahexaenoic acids, respectively EPA and DHA, is associated with a significant reduction in inflam- mation, by means of a reduction of proinflammatory eicosanoids production deriving from arachidonic acid. ${ }^{50-52}$ Spices like turmeric, garlic, curry, prebiotics like inulin, coloured berries, vegetables as well as olive-derived foods (containing lignans) also contribute to an anti-inflammatory and anti-edema action

Among the foods, which conversely generate edema, salt, hydrogenated fats/trans omega 6 or 9, coffeine, theobromine, alcohol, dairies, cereals, sauces, processed meat, junk food, sweets have been shown to increase inflammation and edema through different mechanisms.

Excess sodium tends to retain water in the blood vessels, increases arterial pressure, imbalances microcirculatory homeodynamics and lymphatic function, which subsequently worsens edema. ${ }^{53}$ Consequently the limitation of sodium and foods that contain high doses of this mineral are recommended in lymphedematous patients. There are foods that promote edema through the variation of the endocrine system, such as soy. Soy-based food contains phytoestrogens such as genistein, which has a structure similar to natural estrogens and binds to $\alpha$ - and $\beta$-estrogen receptors to stimulate transcriptional activity. ${ }^{54,55}$ Apart from the ingredients naturally contained in foods, a special attention is to be given to the vast panorama of food additives; many of these, for example carrageenan and glutamate, have notoriously a pro-inflammatory action. ${ }^{56,57}$ Other proedema chemicals are represented by estrogen-like endocrine disrupting chemicals (EEDC), which alter endocrine system functionality and interfere with the synthesis, metabolism, binding or cellular responses of natural estrogens. EEDCs, such as ftalates, bisphenol, glyphosate, have been found in various plastic products, pesticides and many other daily used products. ${ }^{58}$ Also negative interaction with gut microbiota/microbioma and mitochondrial death have been linked to the exposure to most substances cited above, with obvious repercussions on the microvascular/tissue homeostasis. Interestingly a diet rich in fiber may reduce the absorption of estrogens. ${ }^{59}$

Nutrition has proven to interfere significantly with any systemic and loco-regional inflammation, edema, tissue degeneration in general and, ultimately, with metabolism and aging processes. LYM itself is characterised by a chronic inflammatory state that actively involves the extracellular matrix, which leads to the need of a proper nutritional strategy in the holistic view of LYM treatment. More than a calculation of weight and kilocalories, nutrition is intend- ed as a correct and balanced intake of food and nutrients during the day. Any antiinflammatory diet includes a drastically reduced intake of refined carbohydrates, redistributing portions of fats with a prevalence of essential monounsaturated and polyunsaturated fatty acids (more omega 3 and less omega 6) and raising the daily intake of antioxidants as polyphenols.

Proper redistribution of macronutrients during meals allows a better control of the hormonal response (insulin, glucagon, cortisol, estrogen), which in turn results in a significant reduction of the chronic cellular inflammatory state. ${ }^{60}$ Additional outcomes of an anti-s balanced nutrition are weight reduction, improvement in tissue oxygenation. ${ }^{60}$ and ultimately improved glycemic balance. Reduced amounts of refined carbohydrates (insulin stimulators, hence lipogenesis and fluid-retention stimulators) and increased supply of fibers supply, which also improves gut microbiota, have ultimately an anti-inflammatory and antiedema action.

Dietary protein intake does not necessarily correlate with the protein excess in the interstitial matrix, hence a proper intake of protein quota is recommended to synthesize the essential amino acids in LYM patients as well. ${ }^{61}$ High quality organic protein (unprocessed lean red meat, possibly from grass-fed animals, organic white meat and especially small size fish) are usually favoured.

Fats may represent an issue in patients with LYM, who are often overweight or obese and who may get problems from a dense, fat-rich lymph from the intestine. In secondary LYM of the lower limbs, for example subsequent to abdominal-pelvic lymphadenectomy, medium-chain fatty acids (such as coconut oil and some seeds) are preferable, as the long-chain ones being absorbed in the intestinal mucosa may worsen the impaired lymph drainage at intestine level furthermore. The ultimate result of this overloading long fatty chains may be an alteration of the intestinal mucosa, which consequently deteriorates gut inflammation and permeability (leaky gut syndrome). ${ }^{62}$ Conversely, short/medium-chain fatty acids are preferable, ${ }^{43,44}$ as they do not require the secretion of bile salts for digestion and secondly they have the ability to passively pass from the gastrointestinal tract to the blood portal system, thus not overloading the intestinal mucosa.

Hydration balance in LYM disease is considered basic to maintain tissues fluid homeostasis. Coffee and alcohol have both a mild diuretic power which may lead to a protein concentration in the interstice, and may generate edema as well. 


\section{Fasting and lymphedema}

Fasting is another popular form of nutrition strategy to favour weight loss, but even more to reduce chronic inflammatory diseases and LGCCI. More specifically intermittent fasting (IF) is gaining scientific evidence in metabolism literature and more generally in the therapeutic approach to chronic diseases. It has been established that regular intermittent fasting (i.e. 24 hour fasting once or twice a week, or preferably 16 hour fasting most days of the week) confers similar health benefits and weight loss than regular caloric restriction diets. ${ }^{63}$ Moreover in controlled studies when intermittent fasting and standard low calories diet are compared, IF shows higher fat mass loss and improved insulin and leptin reduction, even if the caloric deficit is the same in both protocols. ${ }^{64}$ The mechanisms involve a metabolic shift to fat metabolism and ketone production and stimulation of adaptive cellular stress responses that prevent and repair molecular damage.

Basically IF in lymphedematous patients may not only contribute to obesity/overweight reduction, but also it may represent a key strategy to improve inflammation and regulate altered autoimmune response. Local tissue inflammation involves hyper-activation of macrophages which produce pro-inflammatory cytokines (TNF, IL-1 $\beta$, IL-6) and reactive oxygen species. These processes have been shown as overexpressed in presence of LYM, as well as overweight and obesity clearly promote inflammation. IF was shown to suppress inflammation in human subjects and animals ${ }^{65}$ and multiple studies have shown that fasting can lessen symptoms in patients with immunitary diseases. ${ }^{66}$

The nutrient-responsive mTOR pathway overstimulates protein metabolism but also it negatively regulates autophagy, which may impair tissue natural clearance. Accordingly, fasting inhibits the mTOR pathway and stimulates autophagy in cells of many tissues, including liver, kidney, and skeletal muscle. ${ }^{67}$ Autophagy mechanisms may play a role in the continuous remodelling processes of LYM tissues where necrosis, inflammation and other negative metabolic pathways are chronically activated. ${ }^{68}$ There are several others molecular mechanisms underlying the beneficial effects of fasting on LGCCI and mitochondrial biogenesis, including $\mathrm{NrF} 2$ activation $^{69}$ and anti-oxidant systems, which is of interest in the oxidative stress of lymphedematous tissues. Lastly sirtuins activation in IF positively interferes with apoptosis, autophagy and inflammatory processes in adipose tissue and more broadly in cellular life. ${ }^{70}$

In conclusion the systemic effect of fasting on weight excess, insulin resistance, LGCCI and autoimmunity is to be re-considered in LYM management, in order to possibly include time-restricted feeding in a proper nutrition strategy.

\section{Conclusions}

Nutrition is one of the basic components in the therapeutic management of vascular diseases. LYM is characterised by an increase of interstitial fluid due to morphological and/or functional disease of the lymphatic system. Treatment of LYM is based on multiple components in a holistic and multi-faceted approach, mainly using compression and physiotherapy. The proven importance of weight control and of antiinflammatory strategies in any chronic degenerative disease should be recognized in LYM treatment as well, in a sort of translational medicine application.

This narrative review has highlighted some literature data and information about nutrition and LYM, though the level of scientific evidence for the current knowledge is quite low, due to the lack of robust studies. The intuitive and proven role of nutrition in weight control and in combating inflammatory processes impose a reappraisal of the current therapeutic approach to LYM, so to include a series of possible nutritional measures to fight this chronic degenerative disease. Some preliminary data and our personal experience indicate that edema and chronic inflammation may be controlled through a balanced intake of macronutrients and polyphenols; additional interesting nutritional measures are represented by the introduction of time-restricted feeding (IF) periods, favouring and avoiding proper/wrong foods and additives respectively.

Future randomized studies may elicit the role of several specific foods/nutraceuticals and diets/ fasting regimes in LYM management, so to corroborate the limited literature evidence and finally to improve the necessarily holistic approach to the pathological complexity of the patient affected by this chronic and debilitating disease.

\section{References}

1. The diagnosis and treatment of peripheral lymphedema: 2016 consensus document of the International Society of Lymphology. Lymphology 2016;49:
170-84.

2. Levick JR, Michel CC. Microvascular fluid exchange and the revised Starling principle. Cardiovasc Res 2010;87:198210.

3. Lee BB, Andrade M, Antignani PL, et al. International Union of Phlebology. Diagnosis and treatment of primary lymphedema. Consensus document of the International Union of Phlebology (IUP). Int Angiol 2013;32:541-74.

4. Franceschi C, Bonafe' $\mathrm{M}$, Valensin $\mathrm{S}$, et al. Inflamm-aging. An evolutionary perspective on immunosenescence. 2000. Ann N Y Acad Sci 908:244-54.

5. Thorburn AN, Macia L, Mackay CR. Diet, metabolites, and "westernlifestyle" inflammatory diseases. Immunity 2014;40:833-42.

6. Raffoul W, Far MS, Cayeux MC, Berger MM. Nutritional status and food intake in nine patients with chronic lowlimb ulcers and pressure ulcers: importance of oral supplements. Nutrition 2006;22:82-8.

7. Molnar JA, Vlad LG, Gumus T. Nutrition and Chronic Wounds: Improving Clinical Outcomes. Plast Reconstr Surg 2016;138:71S-81S.

8. Barber GA, Weller CD, Gibson SJ. Effects and associations of nutrition in patients with venous leg ulcers: A systematic review. J Adv Nurs 2018;74: 774-8.

9. Gaffney M, Casley-Smith JR. Excess plasma proteins as a cause of chronic inflammation and lymphoedema. Quant Electr Microscop 1981:229-42.

10. Rockson S. The lymphatics and the inflammatory response: lessons learned from human lymphedema. Lym Res Biol 2013;11:117-20.

11. Ghanta S, Cuzzone DA, Torrisi JS, et al. Regulation of inflammation and fibrosis by macrophages in lymphedema. Am J Physiol Heart Circ Physiol 2015;308: H1065-77.

12. Cuzzone DA, Weitman ES, Albano NJ, et al. IL-6 regulates adipose deposition and homeostasis in lymphedema. Am J Physiol Heart Circ Physiol 2014;306: H1426-34.

13. Rockson SG, Tian W, Jiang X, et al. Pilot studies demonstrate the potential benefits of antiinflammatory therapy in human lymphedema. JCI Insight 2018;3:20

14. Chakraborty S, Zawieja S, Wang W, et al. Lymphatic system acts as a vital link between metabolic syndrome and inflammation. Ann NY Acad Sci 2010;1207:E94-102.

15. Siems WG, Brenke R, Beier A, Grune T. Oxidative stress in chronic lym- 
phoedema. QJM 2002;95:803-9.

16. Krzyściak W, Kózka M. Generation of reactive oxygen species by a sufficient, insufficient and varicose vein wall. Acta Biochim Pol 2011;58:89-94.

17. Krzyściak W, Kózka M, Kazek G, Stępniewski M. Selected indicators of the antioxidant system in the blood of patients with lower limb varicose veins. Acta Angiologica 2009;15:10-9.

18. Glowinski J, Glowinski S. Generation of reactive oxygen metabolites by the varicose vein wall. Eur J Vasc Endovasc Surg 2002;23:550-55.

19. Budzyń M, Iskra M, Krasiński Z, et al. Serum iron concentration and plasma oxidant-antioxidant balance in patients with chronic venous insufficiency. Med Sci Monit 2011;17:719-27.

20. Siti HN, Kamisah Y, Kamsiah J. The role of oxidative stress, antioxidants and vascular inflammation in cardiovascular disease (a review). Vascul Pharmacol 2015;71:40-56.

21. Goszcz K, Duthie GG, Stewart D, et al. Bioactive polyphenols and cardiovascular disease: chemical antagonists, pharmacological agents or xenobiotics that drive an adaptive response? $\mathrm{Br} \mathrm{J}$ Pharmacol 2017;174:1209-25.

22. Calabrese V, Cornelius C, DinkovaKostova AT, et al. Cellular stress responses, hormetic phytochemicals and vitagenes in aging and longevity. Biochim Biophys Acta 2012;1822:75383.

23. Cesarone MR, Belcaro G, Rohdewald P, et al. Improvement of signs and symptoms of chronic venous insufficiency and microangiopathy with Pycnogenol ${ }^{\circledR}$ : A prospective, controlled study. Phytomedicine 2010;11:835-39.

24. Sano A, Tokutake S, Seo A. Proanthocyanidin-rich grape seed extract reduces leg swelling in healthy women during prolonged sitting. J Sci Food Agric 2013;93:457-62.

25. Rabe E, Guex J-J, Morrison N, et al Treatment of chronic venous disease with flavonoids: recommendations for treatment and further studies. Phlebology 2013;28:308-19.

26. Casley-Smith JR, Wang CT, CasleySmith JR, Zi-hai C. Treatment of filarial lymphoedema and elephantiasis with 5,6-benzo-alpha-pyrone (coumarin). BMJ 1993;307:1037-41.

27. Burgos A, Alcaide A, Alcoba C, et al Comparative study of the clinical efficacy of two different coumarin dosages in the management of arm lymphedema after treatment for breast cancer. Lymphology 1999;32:3-10.

28. Roh K, Kim S, Kang H, et al. Sulfuretin has therapeutic activity against acquired lymphedema by reducing adipogenesis. Pharmacol Res 2017;121:230-9.

29. Thangapazham RL, Sharad S, Maheshwari RK. Skin regenerative potentials of curcumin Biofactors 2013;39:141-9.

30. Nguyen TA, Friedman AJ. Curcumin: a novel treatment for skin-related disorder. J Drugs Dermatol 2013;12:1131-7.

31. He Y, Yue Y, Zheng X, et al. Curcumin, inflammation, and chronic diseases: how are they linked? Molecules 2015;20:9183-213.

32. Greene AK, Grant FD, Slavin SA. Lower-Extremity Lymphedema and Elevated Body-Mass Index. N Engl J Med 2012;366:2136-37.

33. Piller N. Links between BMI and the increasing incidence/prevalence of chronic oedema: what is our future? J Lymph 2016;11:1.

34. Davies HO, Popplewell M, Singhal R, et al. Obesity and lower limb venous disease - The epidemic of phlebesity. Phlebology 2017;32:227-33.

35. Greene AK, Grant FD, Slavin SA, Maclellan RA. Obesity-induced lymphedema: clinical and lymphoscintigraphic features. Plast Reconstr Surg 2015;135:1715-9.

36. Mehrara BJ, Greene AK. Lymphedema and obesity: is there a link? Plast Reconstr Surg 2014;134:154e-60e.

37. Ribeiro Pereira ACP, Koifman RJ. Bergmann Incidence and risk factors of lymphedema after breast cancer treatment: 10 years of follow-up. Breast 2017;36:67-73.

38. Ridner SH, Dietrich MS, Stewart B, Armer JM. Body mass index and breast cancer treatment-related lymphedema. Supp Care Cancer 2011;19:853-57.

39. Bertelli G, Venturini M, Forno G, et al. An analysis of prognostic factors in response to conservative treatment of postmastectomy lymphedema. Surg Gynecol Obstet 1992;175:455-60.

40. Fife CE, Benavides S, Otto G. Morbid obesity and lymphedema management. LymphLink 2007;19:1-3.

41. McNeely ML, Peddle CJ, Yurick JL, et al. Conservative and dietary interventions for cancer-related lymphedema: a systematic review and meta-analysis. Cancer 2011;117:1136-48.

42. Shaw C, Mortimer P, Judd PA. Randomized controlled trial comparing a low-fat diet with a weight-reduction diet in breast cancer-related lymphedema. Cancer 2007;109:1949-56.

43. Oliveira J, César TB. Influence of complex decongestive physical therapy associated with intake of medium-chain triglycerides for treating upper-limb lymphedema. Rev Bras Fisioter 2008; 12:31-6.

44. Soria P, Cuesta A, Romero $\mathrm{H}$, et al. Dietary Treatment of Lymphedema by Restriction of Long-Chain Triglycerides. Angiol 1994;45:703-7.

45. Bihun JA, McSherry J, Marciano D. Idiopathic edema and eating disorders: evidence for an association. Int $\mathrm{J}$ Eat Disord 1993;14:197-201.

46. Serino A, Salazar G. Protective Role of Polyphenols against Vascular Inflammation, Aging and Cardiovascular Disease. Nutrients 2019;11:53.

47. Gul K, Singh AK, Jabeen R. Nutraceuticals and Functional Foods: The Foods for the Future World. Crit Rev Food Sci Nutr 2016;56:2617-27.

48. Calabriso N, Massaro M. Extra virgin olive oil rich in polyphenols modulates VEGF-induced angiogenic responses by preventing NADPH oxidase activity and expression. J Nutr Biochem 2016;28:19-29.

49. Meijer K, De Vos P, Priebe MG. Butyrate and other short-chain fatty acids as modulators of immunity: what relevance for health? Curr OpClin Nutr Met Care 2010;13:715-21.

50. Poudyal H, Panchal SK, Diwan V, Brown L. Omega-3 fatty acids and metabolic syndrome: effects and emerging mechanisms of action. Prog Lipid Res 2011;50:372-87.

51. Santos S, Oliveira A, Lopes C. Systematic review of saturated fatty acids on inflammation and circulating levels of adipokines. Nutr Res 2013;33:687-95.

52. Brown L, Poudyal H, Panchal SK. Functional foods as potential therapeutic options for metabolic syndrome. Obesity Rev 2015;16:914-41.

53. Mizuno R, Isshiki M, Ono N, et al. A high salt diet differentially modulates mechanical activity of afferent and efferent collecting lymphatics in murine iliac lymph nodes. Lymphat Res Biol 2015;13:85-92.

54. Nikov GN, Hopkins NE, Boue S, Alworth WL. Interactions of dietary estrogens with human estrogen receptors and the effect on estrogen receptorestrogen response element complex formation. Environ Health Perspect 2000;108:867-72.

55. Cassidy A. Potential tissue selectivity of dietary phytoestrogens and estrogens. Curr Opin Lipidol 1999;10:47-52.

56. Nantel F, Denis D, Gordon R, et al Distribution and regulation of cyclooxygenase-2 in carrageenaninduced inflammation. BJP 1999;128: 


\section{3-59.}

57. Nakanishi Y, Tsuneyama K, Fujimoto $\mathrm{M}$, et al. Monosodium glutamate (MSG): A villain and promoter of liver inflammation and dysplasia. $\mathrm{J}$ Autoimmun 2008;30:42-50.

58. Roy JR, Chakraborty S, Chakraborty TR. Estrogen-like endocrine disrupting chemicals affecting puberty in humansa review. Med Sci Monit 2009;15:RA 137-45.

59. Rose DP, Goldman M, et al. High-fiber diet reduces serum estrogen concentrations in premenopausal women. Am J Clin Nutr 1991;54:520-5.

60. Sear B. Zone perfect meal. New York: Regan Book; 1998.

61. Dawson R, Piller N. Diet and BCRL: facts and fallacies on the web. J Lym 2016;1:36-42.

62. Fasano L. Gut and autoimmune disease. Clin Rev All Immun 2010;42:71-8.

63. Barnosky AR, Hoddy KK, Unterman
TG, Varady KA. Intermittent fasting vs daily calorie restriction for type 2 diabetes prevention: a review of human findings. Transl Res 2014;164:302-11.

64. Kahleova H, Belinova L, Malinska H, et al. Eating two larger meals a day (breakfast and lunch) is more effective than six smaller meals in a reducedenergy regimen for patients with type 2 diabetes: a randomised crossover study. Diabetologia 2014;57:1552-60.

65. Kroeger CM. Improvement in coronary heart disease risk factors during an intermittent fasting/calorie restriction regimen: relationship to adipokine modulations. Nutr Metab 2012;9:98.

66. Müller H, de Toledo FW, Resch KL. Fasting followed by vegetarian diet in patients with rheumatoid arthritis: A systematic review. Scand J Rheumatol 2001;30:1-10.

67. Vendelbo MH, Møller AB, Christensen $\mathrm{B}$, et al. Fasting increases human skele- tal muscle net phenylalanine release and this is associated with decreased mTOR signaling. PLoS One 2014;9:e102031.

68. Zaleska MD, Olszewski W. Serum Immune Proteins in Limb Lymphedema Reflecting Tissue Processes Caused by Lymph Stasis and Chronic Dermatolymphangioadenitis (Cellulitis). Lym Res Biol 2017;15:3.

69. Supriya RK, Donepudi AC, Jialin Xu, et al. Fasting Induces Nuclear Factor E2Related Factor 2 and ATP-Binding Cassette Transporters via Protein Kinase A and Sirtuin-1 in Mouse and Human. Antioxid Redox Signal 2014;20:15-30.

70. Wegman MP, Guo MH, Bennion DM, et al. Practicality of Intermittent Fasting in Humans and its Effect on Oxidative Stress and Genes Related to Aging and Metabolism. Rejuvenation Res 2015; 18:162-72. 